\title{
Germanica
}

\section{Pour en finir avec les pères ? La nouvelle de Friedrich Christian Delius Amerikahaus und der Tanz um die Frauen}

Um die Väter endgültig zu vergessen? Die Novelle von Friedrich Christian Delius "Amerikahaus und der Tanz um die Frauen"

\section{Alain Cozic}

\section{OpenEdition}

\section{Journals}

Édition électronique

URL : http://journals.openedition.org/germanica/2159

DOI : 10.4000/germanica. 2159

ISSN : 2107-0784

Éditeur

Université de Lille

\section{Édition imprimée}

Date de publication : 30 juin 2002

Pagination : 101-116

ISBN : 9782913857070

ISSN : 0984-2632

Référence électronique

Alain Cozic, «Pour en finir avec les pères ? La nouvelle de Friedrich Christian Delius Amerikahaus und der Tanz um die Frauen », Germanica [En ligne], 30 | 2002, mis en ligne le 16 juillet 2013, consulté le 06 octobre 2020. URL : http://journals.openedition.org/germanica/2159; DOI : https://doi.org/10.4000/ germanica. 2159

Ce document a été généré automatiquement le 6 octobre 2020.

(c) Tous droits réservés 


\title{
Pour en finir avec les pères? La nouvelle de Friedrich Christian Delius Amerikahaus und der Tanz um die Frauen
}

\author{
Um die Väter endgültig zu vergessen? Die Novelle von Friedrich Christian Delius \\ "Amerikahaus und der Tanz um die Frauen "
}

Alain Cozic

1 La nouvelle Amerikahaus und der Tanz um die Frauen (1997) ne peut se comprendre sans celle que F.C. Delius a écrite trois ans auparavant, Der Sonntag, an dem ich Weltmeister wurde. C'est à l'évidence en effet le même personnage que l'on retrouve, même s'il est plus âgé et si les circonstances sont autres. Le jeune garçon de onze ans qui habitait Wehrda, village de la Hesse, est à présent un étudiant de vingt-deux ans à Berlin Ouest. Der Sonntag se passe le 4 juillet 1954, Amerikahaus le 5 février 1966. Dans les deux nouvelles, l'auteur circonscrit donc le segment temporel de son récit, conte, dans un cas comme dans l'autre, certains événements d'une journée particulière dans la vie de son personnage, en 1954 comme en 1966 déterminants pour ce dernier. Les faits relatés sont prolongés et commentés par des réflexions du protagoniste - narrateur s'exprimant à la première personne dans Der Sonntag, narration privilégiant le discours indirect libre dans Amerikahaus - et, dans la seconde nouvelle principalement, par des rappels du passé, remémorations venant s'immiscer dans le présent du narrateur, l'éclairant, lui donnant un relief et une coloration particuliers. Le passé évoqué dans Amerikahaus relatant des événements ou une atmosphère qui constituaient pour partie la trame de Der Sonntag, il convient d'analyser la seconde nouvelle en gardant toujours la première en perspective.

2 C'est ce que les lignes qui suivent se proposent de faire. C'est ce qui explique aussi le titre de cette contribution, dans sa formulation et dans sa forme interrogative. En effet, lorsque s'achève Der Sonntag, le lecteur a le sentiment que le jeune garçon, au centre et narrateur de la nouvelle, sur un point essentiel en tout cas, « en a fini avec les pères ». 
Car il s'agit bien pour le fils de vaincre le père, de surmonter les inhibitions que provoquent sa présence - son omniprésence et son omnipotence -, l'éducation que luimême a reçue et dont il perpétue les principes.

Der Sonntag nous conte l'histoire d'une individualité bridée - celle d'un jeune garçon de onze ans, fils de pasteur-, d'une identité qui, en dépit des souhaits de ceux qui ont en charge l'éducation et qui croient en la validité de principes à leurs yeux irréfragables, ne parvient pas à se constituer. Adolescence en lambeaux donc, qui subit les effets désastreux du dogme quand il devient dogmatisme, qu'il freine, endigue, paralyse tout épanouissement. Mais Delius, simultanément, en imbriquant avec une grande maitrise l'arrière-plan historico-sportif - ce dimanche 4 juillet 1954 qui voit l'équipe nationale de football de la RFA remporter, contre la Hongrie, la finale de la coupe du monde - et la situation psychologique très particulière de son protagoniste, montre aussi comment l'enfant "devient" "champion du monde ", à son échelle, champion du monde des mots, en d'autres termes comment il se pose, s'impose face au père, à l'instance sinon et depuis toujours tant redoutée. On peut considérer qu'une lueur éclaire la fin de cette nouvelle, même si elle est encore vacillante. A partir de ce dimanche, la personnalité fragilisée de l'adolescent acquiert quelque fermeté, les éléments épars d'une psyché disjointe semblent se fondre en un tout cohérent, un individu paraît accéder à luimême ${ }^{1}$. Amerikahaus va démontrer qu'il n'est pas aussi aisé « d'en finir avec les pères ».

\section{Des mots et des maux}

4 Le protagoniste a désormais onze ans de plus, se prénomme Martin (ce qui n'était pas dit dans Der Sonntag), a quitté sa famille et Wehrda, vit à Berlin Ouest, ce qui lui a permis d'échapper au service militaire, est étudiant en germanistique, écrit - nous y reviendrons - à ses heures des poèmes. Il fréquente la jeunesse berlinoise, étudiants et artistes, bohème intellectuelle qui s'exprime d'abondance sur tous les sujets du moment, maniant avec maestria, aux yeux de Martin en tout cas, le langage.

On peut dire que la nouvelle s'organise thématiquement autour de cette notion même de langage. De mots il est en effet de nouveau, comme dans Der Sonntag, beaucoup question dans Amerikahaus. Par touches successives, Delius esquisse l'arrière-plan historique et social sur lequel il inscrit le déroulement de la journée de son personnage. L'un des premiers éléments, au début de la nouvelle, est l'allusion à la mort, quatre jours auparavant, le $1^{\text {er }}$ février 1966, de Buster Keaton. Buster Keaton, l'acteur et le réalisateur américain, dont on se rappelle le nœud papillon mou, l'impassibilité tout autant que le sens du rythme et du gag, caractéristiques qui firent de lui l'un des comiques les plus inventifs et les plus populaires du cinéma muet. Or, saisissant au bond l'événement d'actualité que constitue la mort de Keaton, les étudiants que côtoie Martin le surnomment aussitôt « Buster $»^{2}$. «Buster », parce qu'il est capable, au cours d'une soirée, de ne rien dire ou presque des heures durant, lui « le silencieux ». Certes il ne s'agit que d'une plaisanterie, de ce genre de sobriquets dont les jeunes ont coutume de s'affubler. Mais le surnom désigne bien le laconisme habituel, sinon l'absence de paroles - de mots prononcés - de Martin, traçant ainsi un parallélisme entre cette particularité du caractère du jeune homme et l'impassibilité légendaire de l'acteur du cinéma muet. En outre, cette comparaison n'est pas dénuée d'une certaine ironie, fûtelle involontaire de la part de ceux qui y ont songé : car Keaton était un acteur comique, fondait même son jeu comique sur cette impassibilité qu'il affichait pour 
affronter les aléas des situations où il était plongé. Or, de comique il ne saurait être question à propos de Martin. Plus encore même. À l'instar de ce que faisait le protagoniste de Der Sonntag dans son récit-confession à la première personne, Martin nous livre aussi les réflexions que lui inspire son existence. Celles qu'il formule, lorsque lui est attribué le surnom de "Buster ", sont particulièrement révélatrices ${ }^{3}$. Certes, la distance créée de la sorte par la réflexion - verbalisée en outre - par rapport à cette inhibition qui le traumatise est déjà une manière de surmonter ce blocage : les maux atténués - à défaut d'être supprimés - par les mots qui les disent, la pensée qui formule. En outre, considéré par les autres comme un "solitaire ", " un silencieux », il entre en quelque sorte dans leur jeu dont il pousse jusqu'au bout la logique en transformant l'opinion que les autres ont de lui, la caractéristique qu'ils lui assignent, en rôle qu'il se propose de jouer; accepter d'être ce que les autres font de lui, s'approprier délibérément cette caractérisation par les autres, exploiter cette faiblesse, c'est en somme - ne serait-ce que par la conscience qu'il a du processus mis en œuvre transformer en élément positif, sinon valorisant ce qui, au départ, même s'il ne s'agissait que d'une boutade, ne pouvait être perçu par lui que comme une comparaison désobligeante, mettant à nu et à vif un trait majeur de sa personnalité. Il n'empêche : cette réflexion qui vise à définir un comportement à adopter face aux autres - aux instances qui, l'assimilant ainsi à Buster Keaton, d'une certaine manière le jugent - dit aussi l'individu clivé qu'il est, qui - précisément - devient ce que les autres font de lui. La supériorité que paraissent lui conférer le rôle qu'il joue et la conscience qu'il a de jouer ce rôle n'est qu'apparente : il ne s'agit ici en aucune manière d'une personnalité se construisant de façon autonome.

\section{«Danse entre les femmes », " Danse autour des femmes »}

Ironique, la comparaison avec Buster Keaton l'est encore lorsque sont évoquées les relations, dans les films de l'acteur américain, entre le personnage qu'il joue et les femmes. Ironie d'autant plus amère que dans ce passage aussi l'on a moins affaire à une instance narrative relatant à la troisième personne les pensées du protagoniste qu'à un discours indirect libre dans lequel surgiraient, au moment même où il les formulerait, les propres réflexions de Martin ${ }^{4}$. Comparaison ironique car, dans les films, les femmes aiment le personnage incarné par Keaton malgré, voire à cause, de sa timidité et de ses multiples maladresses, alors que dans la réalité les femmes n'aiment pas Martin - du moins se l'imagine-t-il ainsi. Conscience douloureuse du jeune homme d'échouer aussi - auprès des femmes, lesquelles seraient rebutées par sa laideur, ces femmes au corps - au sexe - mystérieux, inquiétant, dangereux ${ }^{5}$. Les relations de Martin avec les femmes constituent, par rapport à Der Sonntag, l'un des éléments majeurs de la trame narrative; la seconde partie du titre de la nouvelle trouve dans ce motif son explication. La formulation Der Tanz um die Frauen reprend, en le modifiant légèrement mais significativement, le titre d'un tableau peint en 1915 par Ernst Ludwig Kirchner (Der Tanz zwischen den Frauen), tableau aperçu par Martin dans un catalogue que lui avait offert son père à Noël alors qu'il avait seize ans. La peinture de Kirchner représente un homme nu dansant en effet "entre» deux femmes elles aussi nues. Scène des plus énigmatiques même si la relation entre l'homme et la femme, relation de couple partagée entre l'amour et la lutte, traverse l'œuvre de Kirchner et même si la 
position de l'homme et le pas de danse qu'il esquisse ne sont pas sans rappeler une photographie datant de la même époque prise dans l'atelier berlinois du peintre et montrant un homme nu (Kirchner lui-même ?) dansant devant, semble-t-il, une femme debout et un autre homme assis à l'arrière-plan. Si le tableau de Kirchner demeure mystérieux, la nouvelle de Delius en propose une description exacte et surtout une interprétation suggérée par Martin, qui assimile sa propre relation avec les femmes à celle symbolisée sur le tableau par les trois personnages. L'insertion dans la trame narrative de cette description du document iconographique et de l'interprétation qui en est faite est une mise en abîme de la situation de Martin, la reproduction métaphorique de la relation qu'il entretient avec les femmes, voire de son existence même où dominent l'impossible communication avec l'autre, le sentiment d'exclusion et de solitude. Double mise en abîme contradictoire même, ou plus exactement dont la seconde complète et nuance la première au fur et à mesure que Martin interprète : en effet, dans un premier temps, il s'assimile bien à celui qui «danse entre les femmes, entre toutes les femmes ", celles qu'il connaît et vers lesquelles il se sent attiré (Franziska, Ellen, Margret), celles imaginées ou fantasmées (comme celle aperçue pendant la manifestation), qui danse "seul » avec lui-même, mais il est alors celui qui s'est détourné des femmes, qui n'a pas voulu répondre à leurs signes et à leurs appels (c'est une interprétation possible, plausible du tableau de Kirchner); dans un second temps toutefois, à l'inverse de ce que semble suggérer la peinture, il considère que ce sont les femmes qui se sont détournées de lui lorsqu'il s'en est approché, mais pour mieux se reprocher aussitôt sa propre pusillanimité ${ }^{6}$. Le processus ici décrit est, somme toute, analogue à celui étudié à propos de la comparaison avec Buster Keaton: si l'évocation du tableau de Kirchner - remémoration d'un souvenir d'enfance, description détaillée, réflexions induites - offre à Martin l'occasion d'une analyse psychologique précise et lui donne donc la possibilité de penser sa propre situation, elle le renvoie aussi, simultanément, à son isolement, accentuant encore son sentiment d'exclusion, d'auto-exclusion. La prise de conscience que permet la verbalisation n'est pas pour autant libératrice. Dire l'inhibition ne signifie pas la surmonter et la vaincre définitivement ${ }^{7}$. L'introspection d'un moi ne vaut construction d'un je. Et ce d'autant moins que l'observation introspective, lorsqu'elle se conjugue avec une imagination des plus vives, produit parfois des effets peu propices à l'affermissement d'une identité vacillante, soulignant au contraire l'individualité scindée, le dédoublement paranoïde : ainsi ce "dialogue» entre le "corps" et la "tête», l'un reprochant à l'autre sa timidité, les deux se renvoyant mutuellement la " faute $»^{8}$; ainsi encore cette tentative pour s'imaginer ce que les femmes s'imaginent à son propos et ne retenir que ce qui le dessert ${ }^{9}$.

\section{Corps meurtri, corps inquiétant}

7 Der Sonntag insistait sur la desquamation du corps de l'enfant à certains endroits et sur la phobie du sang qui perturbait l'adolescent, Amerikahaus fait de nouveau allusion à ces deux éléments qui, donc, onze ans plus tard, n'ont pas disparu.

Le corps - outre celui des femmes - est, à maints égards, au centre des préoccupations de Martin. Son propre corps qu'il observe, scrute pour en souligner ce qu'il considère être de la laideur, comportement qui était déjà le sien adolescent et qu'à vingt-deux ans il continue d'avoir, comme si se prolongeait l'époque pubertaire, comme si se 
produisaient encore, psychologiquement non surmontées, toutes les modifications physiques et psychiques qu'entraîne ce passage de l'enfance à l'adolescence ${ }^{10}$. Corps malade aussi, portant aux coudes notamment les marques de cette affection dartreuse, cette dermatose qui, autrefois, exposait déjà aux regards des autres le corps meurtri de l'enfant qu'il était et qui, à présent, accroît la honte qu'il a de son corps dénudé ${ }^{11}$. Corps inquiétant et perturbant encore par ses sécrétions, par les liquides qui le traversent et peuvent s'en écouler, ainsi le sang. Phobie du sang qui, dans Der Sonntag, fait s'évanouir l'adolescent lorsqu'un jour est racontée en classe l'histoire d'un jeune hémophile condamné par sa maladie, ce sang qui lui rappelle tout à la fois ses propres saignements de nez et l'image - inculquée depuis toujours par l'éducation religieuse reçue et qui imprègne sa pensée - du Christ ensanglanté sur la croix, ce sang qui le "bouleverse ", ce sang qui avait transformé en échec cuisant sa première expérience sexuelle, épisode aux répercussions psychologiquement désastreuses sur le moment, aux effets inhibiteurs par la suite, qu'inévitablement il se remémore dans Amerikahaus au moment de vivre avec Rahel sa deuxième expérience ${ }^{12}$.

\section{L'ombre portée du passé}

Mais Amerikahaus - et c'est en cela que les liens avec Der Sonntag sont essentiels - ne décrit pas seulement la situation présente de Martin. La nouvelle tisse aussi un réseau d'éléments, rappels du passé, de certaines figures de ce passé et de ce que ces figures ont un jour, dans l'enfance de Martin, représenté. Le passé vaut explication partielle du présent.

10 Car, pour l'étudiant de vingt-deux ans, le passé, précisément, ne passe pas, il couvre, envahit, hante le présent ; souvenirs en aucune manière gommés par le temps mais qui continuent au contraire à marquer le présent de signets ineffaçables. Et de nouveau il est question de mots, et des maux que ceux-ci provoquent. Mots prononcés et inculqués par d'autres. Mots, formules, préceptes édictés et martelés autrefois par ceux qui avaient prise sur sa vie d'adolescent et qui reviennent à sa mémoire, mots faisant surgir des images, marquant telle situation de sa vie d'aujourd'hui. Ainsi la ponctualité tatillonne du grand-père ${ }^{13}$ que contredit l'imprévisibilité - l'un des charmes - de Franziska. Ainsi le sacro-saint respect de l'horaire qui fait que l'on ne s'échappe pas du cortège de manifestants où l'on défile avant la fin comme naguère on ne quittait pas le service religieux avant qu'il ne s'achève ${ }^{14}$. Ainsi l'article de Konkret qu'il lit («Alles über Sex-Partys in den USA ») et à propos duquel le mot " envie ", " désir » ("Lust ») lui vient à l'esprit, terme autrefois tabou, prohibé ${ }^{15}$. Ainsi le film de Pasolini («La Ricotta ») et ses scènes de crucifixion qui lui en rappellent d'autres ${ }^{16}$. Ainsi encore - et de façon générale - le système de règles, de normes qui régissaient la vie, les interdictions qui frappaient ce que l'instance paternelle, elle-même émanation du Père, jugeait impur et relevant du péché. C'est une éducation dans son ensemble - éducation religieuse, protestante, essentiellement - qui est ici évoquée, dont on voit comment elle a façonné, forgé, formé un individu, en réalité déformé et annihilé une personnalité. Gangue paralysante dont il est malaisé de se défaire et dont Martin perçoit avec d'autant plus d'acuité l'emprise qu'il se livre à un acte - aller manifester - qui, par définition, est anormal, acte de résistance, transgression de la règle, qui plus est lorsqu'il s'agit de protester contre la guerre du Vietnam, devant la "Maison de l'Amérique " (justification de la première partie du titre), en RFA et à Berlin, de se révolter contre les 
bombardements en Asie de ceux qui sont pour les Allemands et les Berlinois de l'Ouest "les amis et les protecteurs $»^{17}$. Il est difficile, sinon impossible, de briser «les rets d'anciennes interdictions ", et même lorsque les mentors ont disparu (le père), ils persistent et survivent par l'héritage - les valeurs, autant d'impératifs catégoriques négatifs - qu'ils ont transmis ${ }^{18}$. Il n'est décidément pas aisé d'en finir avec les pères, de se débarrasser d'un legs à ce point pesant où, entre autres éléments constitutifs encore, l'on en appelle à la clause de conscience pour justifier de ne pas se révolter contre l'autorité, quelle qu'elle soit, l'on érige la mauvaise conscience en principe d'éducation, en fondement de l'existence, l'on dénigre et condamne tel domaine ou telle pratique, l'on disqualifie et diabolise le corps tabou comme lieu et réceptacle du péché19, l'on confère à la punition qui suit la faute une vertu éducatrice, ou, pis encore, l'on fait naître un sentiment de culpabilité avant même que la «faute » ne soit commise ${ }^{20}$. Legs d'autant plus oppressant qu'il fait surgir dans l'imaginaire de l'enfant un univers fantasmé dont l'adulte a gardé souvenance, où le père, à l'instar d'Abraham (épisode de Der Sonntag), apparaît le bras armé d'un couteau, couteau qui tranche la langue et rend muet, langue ("Zunge ») coupée et, donc, langue («Sprache ») amputée. Couteau qui tranche le membre. Père qui castre et tue le fils ${ }^{21}$.

11 Interdit et tabou, faute et péché, culpabilité et mauvaise conscience, corps détesté et corps refoulé, honte et angoisse, disphonie et blemmophobie - peur du regard d'autrui -, désarroi face aux femmes, mutisme et solitude: tels sont les éléments autour desquels, en 1966, s'oriente l'existence de Martin. Éléments qui brossent, après celui d'un adolescent dans Der Sonntag, dans Amerikahaus le portrait d'un jeune homme toujours fragilisé dans son intégrité physique et psychique, à l'identité chancelante, introverti, névrotique, velléitaire, qui font de lui, aux yeux des autres et à ses propres yeux, "celui qui parle peu, boit peu et part tôt ", qui "échoue face aux femmes", "l'étudiant muet, le manifestant gauche, l'amoureux maladroit "22. Traits physiques et psychologiques dont les conséquences pour son existence sont d'autant plus fortes qu'à vingt-deux ans la conscience qu'il a de semblables handicaps est plus aiguë. Et si l'on considère le prénom comme l'un des premiers traits fondateurs d'une identité - ténu certes mais parfois, et en l'occurrence, déterminant - force est de constater que celui qui lui a été donné ne contribue guère à affermir sa personnalité : « Martin »... comme Luther, avec tout ce qu'implique pour lui, en matière de dogmatisme religieux, l'allusion au Réformateur. Prénom lui aussi refuséé ${ }^{23}$.

La manifestation de protestation contre la guerre du Vietnam est encore pour Martin l'occasion de se livrer à l'une de ces réflexions dont il est coutumier - ces «autoanalyses ", ces bilans psychologiques auxquels, au fil de la nouvelle, il ne cesse de se livrer, disent du reste assez ses préoccupations -, et qui montre bien une nouvelle fois les contradictions qui le tourmentent, la conscience clivée qui est la sienne. Il est en effet partagé entre le désir de ne rien faire allant contre la règle pour ne pas être fautif et puni - or, manifester, à cette époque et pour quelqu'un comme Martin, ne peut être perçu que comme transgression de l'ordre - et la crainte, en restant passif et silencieux, d'être le complice d'une guerre meurtrière qu'il réprouve par ailleurs ${ }^{24}$. Si l'on assiste aussi, dans cette nouvelle, à la naissance d'une conscience politique, celle-ci ne se fait pas sans difficultés ni tâtonnements. Doutes et atermoiements, interrogation térébrante de la conscience qui le font hésiter entre deux attitudes contradictoires, inconciliables: lancer des œufs contre la façade de la «Maison de l'Amérique » et 
mettre le drapeau américain en berne, ou assister, en simple témoin, à la manifestation. Intervenir ou observer. Agir ou se dérober.

\section{Agir, réagir}

13 Agir, réagir pour Martin : telle est bien en effet, comme dans Der Sonntag déjà, mais ici beaucoup plus développée, l'autre composante majeure de Amerikahaus. Formulées sous forme d'interrogations, ses réflexions au sujet de l'éducation qu'il a reçue, du rôle du père et du grand-père, si elles disent à quel point il est enfermé dans ce qui - en vertu des principes enseignés - doit être, montrent aussi, simultanément, comment il perçoit ce qui pourrait/devrait être ${ }^{25}$. Particulièrement significatif est ici le terme "Ausschreitungen » qui sera repris, dans une autre réflexion à la fin de la nouvelle, repris et varié, modulé, substantif défini, verbe conjuguée ${ }^{26}$. Car c'est bien de cela qu'il s'agit : ne plus « marcher droit », ne plus « marcher aux ordres », choisir les chemins de traverse plutôt que la voie officielle, droite, balisée, normée, échapper à la norme, dévier. En d'autres termes : ne plus exister au nom du père et du grand-père, au nom du Père, du diable et du bon Dieu, du Bien et du Mal. Ou encore, et fondamentalement: construire sa singularité et non plus la voir asservie à quelque système qui la bride et la brise, à l'hétéronomie d'un moi assujetti substituer l'autonomie d'un je affirmé.

14 Sa participation, ce jour-là à Berlin, au cortège de protestation contre la guerre du Vietnam est-elle, à cet égard, une manifestation de son indépendance, un acte de résistance ? La réponse est ambiguë. Elle est révolte dans la mesure où, ce faisant, il se pose en s'opposant à ceux qui ne manqueraient pas de réprouver une telle action, son père s'il vivait encore, son grand-père assurément, sa mère aussi. Elle l'est aussi d'une certaine manière, parce qu'en manifestant contre cette guerre, il refuse toute lutte armée, lui l'enfant de la guerre - dont les séquelles ont tué son père après que le conflit précédent eut emporté le père de celui-ci - et de l'après-guerre ${ }^{27}$. Mais peut-on parler encore de révolte dès lors que participer à cette manifestation (fort pacifique encore nous ne sommes qu'en 1966 -, où des étudiants disciplinés défilent en respectant les directives de policiers portant cravate) est - aussi - ressenti par ce jeune homme lucide comme un acte dicté par les circonstances, qui le fait se conformer, lui qui n'appartient à aucun groupe ni parti, à une attitude qu'aux yeux de certains il est de bon ton d'avoir ${ }^{28}$ ?

Peut-on parler d'inhibition surmontée lorsque la foule des manifestants est tout à la fois perçue comme un cadre rassurant et protecteur (personne dans cette foule ne l'observe, lui, en particulier : blemmophobie, toujours) et lieu où il faut se montrer en public et donc s'exposer ${ }^{29}$ ?

Agir, réagir contre le trouble de langage qui le perturbe. La victoire, dans Der Sonntag, sur les combinaisons de consonnes impossibles à prononcer a été aussi brutale que ponctuelle (le «zwei zu zwei » articulé sans difficultés devant son père à la mi-temps du match retransmis à la radio). Systématique est, dans Amerikahaus, le processus mis en œuvre. Il s'agit bien en effet d'une méthode systématiquement appliquée, d'une manière de stratégie d'évitement : faire en sorte de n'avoir pas à prononcer les mots ou les ensembles de lettres qui sont autant de pièges, les occlusives par exemple ${ }^{30}$. Certes est évoquée ici une activité mentale, cérébrale intense qui lui permet de circonscrire comme des espaces d'infléchissement, des micro-intervalles de liberté insérés dans une réalité - la disphonie - par ailleurs inhibante et aliénante. Mais l'effort nécessaire pour 
mettre en œuvre ce processus compliqué ne peut, à l'évidence, que renforcer encore la gêne. Les victoires sur les mots rétifs ne sauraient être que provisoires et imparfaites. En outre, les termes qu'il est contraint d'utiliser pour remplacer ceux qu'il ne peut prononcer sont parfois moins exacts ${ }^{31}$. La substitution est donc approximative, sinon erronée. Les mots ne sont plus que la traduction infidèle des pensées, tout développement cohérent d'une certaine ampleur devient de la sorte impossible. Malgré la volonté et les efforts accomplis, il se retrouve en définitive relégué dans son rôle de «muet ». Buster, décidément, n'est pas un héros du cinéma parlant!

Toutefois, si Martin ne parvient pas à produire un discours oral à la hauteur de ses espérances, de ses capacités intellectuelles et de son imagination, il excelle en revanche dans l'écriture, le texte écrit devient ce que la parole ne peut être, la langue ("Sprache ») sera tout de même sauvée lorsque la langue ("Zunge ») fera défaut.

Lecteur passionné, Martin l'est parce que ses études l'exigent, mais aussi et surtout parce que les livres qu'il lit sont un refuge, une source qui nourrit son imagination, le langage imprimé des autres lui fait oublier ses propres carences ${ }^{32}$. Ces mêmes études font que lui aussi, parfois, doit écrire, quand il lui faut préparer un exposé par exemple. Mais outre ces "travaux de commande », il écrit aussi pour lui-même, par plaisir ou nécessité, des poésies notamment. Avec le texte écrit qu'il produit, il ne bafouille plus, les touches de la machine à écrire qu'il utilise supplantent la langue qui fourche, les mots qui naissent ainsi au bout des doigts ont l'assurance et la fermeté que n'ont pas ceux que parfois - souvent - sa bouche articule avec peine. Être en écrivant donc, même si cela se fait par machine interposée, et même si l'acte s'accomplit dans la solitude de sa chambre d'étudiant. Mais précisément: dans cet espace clos, il ne sent plus, derrière lui, peser sur lui le regard qui scrute et juge, celui du père, du grand-père, de la mère, de Dieu ${ }^{33}$. La solitude de sa chambre, en somme, pour échapper à l'isolement où le confinent par ailleurs ses inhibitions. Mais, dès lors, cette nouvelle forme d'enfermement vaut-elle accession à lui-même, réalisation d'une identité assurée ? Ses réflexions à propos de l'exposé qu'il doit faire disent bien, de nouveau, les sentiments contradictoires qui l'assaillent, ses difficultés extrêmes à être au monde ${ }^{34}$. Car, de nouveau et toujours, c'est bien de cela qu'il s'agit: de la question ontologique de son existence. Attirer l'attention des autres étudiants par l'originalité de son exposé, en d'autres termes être par l'écriture ce qu'il ne peut être par ailleurs, ne plus passer inaperçu, briser l'anonymat, susciter la sympathie, fût-ce au prix d'une certaine fatuité dont il a du reste conscience ${ }^{35}$.

Mais l'acte d'écriture lui-même, même - surtout - lorsqu'il ne s'agit pas d'un travail imposé (un exposé à faire en cours) mais d'une création originale et des plus personnelles (un poème), n'est pas exempt de certains dangers. Les mots qui viennent à l'esprit et que transcrivent les doigts actionnant les touches de la machine viennent aussi parfois de loin, de l'enfance, de l'éducation reçue, ils ont à ce point imprégné le subconscient que machinalement ils se manifestent alors que, précisément, il faudrait les bannir ou ne les utiliser tout au plus qu'avec la distance salvatrice qu'installe l'ironie. Ainsi "Leib», le "mot du père ", le "mot étranger ", aux connotations chrétiennes, marqué du sceau du tabou ${ }^{36}$. Et la violence des termes ici utilisés dit assez l'influence subie, perçue comme néfaste, paralysante, montre la contamination par une langue aux yeux de Martin dévoyée venue soudain paralyser un langage qu'il pensait libéré. Il n'est pas aisé, vraiment, d'en finir avec les pères ! 
20 Agir, réagir contre le corps dont on a honte et qu'on méprise. Au cours de la journée évoquée dans Der Sonntag, la radio a joué un rôle essentiel. De la même manière ici, les émissions que Martin écoute dans sa chambre quand il écrit - musique de jazz par exemple - font entrer dans la pièce le monde extérieur qui, cette fois, n'est pas perturbant mais, à l'instar de ce qui était décrit dans Der Sonntag, libérateur. Ainsi la musique de Charlie Mingus, contrebassiste et pianiste, le grand jazzman des années soixante, le virtuose à la dextérité peu commune, le styliste parfait, mais aussi celui qui déconstruit les standards. Ainsi l'instrumentiste remarquable qu'est Stan Getz, le saxophoniste à la sonorité aérienne, celui que l'on surnomma dès les années cinquante "The Sound ». Autrement dit: deux musiciens exceptionnels, maîtres de leur art, au langage musical à même d'exprimer toutes les nuances de leur sensibilité, deux artistes qui ne "bafouillent " pas. Révélateur est le passage où, au son de "East of the sun and west of the moon » de Stan Getz, Martin, dans sa chambre, se met à danser. Nouvelle variation sur le thème de la danse, après le tableau de Kirchner, danse-transe qui permet au corps de s'exprimer enfin, de s'extraire de la gangue des principes et des préceptes délétères, des commandements et des interdits mortifères, corps naguère peccamineux, s'affirmant à présent, se sculptant. Certes il danse seul, mais cette solitude, à cet instant, est libératrice.

21 De danse il est encore question - autre variation sur le thème - après qu'il a rencontré par hasard dans la boîte de nuit «Big Apple» Rahel, l'Israélienne. On peut dire que l'épisode de la rencontre avec la jeune femme, à la fin de la nouvelle, reprend et condense les thèmes de celle-ci, en même temps qu'il propose une réponse aux énigmes qui marquent l'existence de Martin. Au «Big Apple», lorsqu'il fait sa connaissance, Rahel, elle aussi, danse seule : deux solitudes face à face, qui se rejoignent bientôt, après que les regards se sont croisés et que Rahel a pris l'initiative de lui saisir la main. Les pensées de Martin, à cet instant, en pareilles circonstances, disent comment cette danse-là aussi est libératrice, comment à l'image du danseur isolé, replié sur lui-même, comme paralysé, figé de Kirchner tente de se superposer celle du danseur à présent épanoui et heureux qu'il a le sentiment d'être ${ }^{37}$. Non plus «Danse autour ou entre les femmes » mais bien « danse avec une femme ».

Rahel, l'Israélienne de Tel Aviv venue à Berlin vanter les mérites de l'orange et du pamplemousse lors de l'immense manifestation qu'est la « Grüne Woche », Rahel qui ne s'exprime avec Martin qu'en anglais, langue que le jeune homme parle aussi, même si ce n'est «pas très bien ${ }^{38}$, mais langue qui n'est plus la langue allemande avec tout ce que celle-ci comporte de blocages, de troubles émotionnels pour Martin, langue qui permet l'expression libre, même si des hésitations subsistent, qui offre surtout la possibilité de communiquer avec l'autre. Rahel, de passage à Berlin, la femme libre, libérée, qui, à l'évidence, ne subit pas le poids d'interdits ancestraux, qui lui propose, sans ambages, de passer la nuit avec elle. Si, à cette idée, les vieux démons resurgissent, le souvenir du sang qui, un jour, s'était mis soudain à couler à flots de son nez, si l'expérience se révèle pour Rahel des plus décevantes, l'acte, pour Martin, est - d'une certaine manière - libérateur. Le corps de Rahel, en effet, lui révèle son corps, lui permet de briser les tabous prononcés par le père, le Père, sur le corps, d'en finir avec la - mauvaise - conscience ; cette nuit-là c'est moins son innocence qu'il perd qu'un sentiment de culpabilité, de honte, de péché qu'il portait depuis toujours chevillé en lui ${ }^{39}$. Les dernières paroles que lui adresse Rahel («Take it easy») valent 
encouragement, sont comme une maxime qui, désormais, pourrait orienter son existence.

« Image d'une jeunesse ». L'on voit comment cette nouvelle de Delius s'organise autour d'un faisceau d'éléments convergents, distribués sur un arrière-plan évoquant l'actualité sous de multiples formes à Berlin et dans le monde en 1966 : solitude d'un être, repli sur soi, inhibitions, troubles du langage, corps honteux, sexualité perturbante, peur de la femme, ombre écrasante des pères et des principes inculqués, assénés ; mais aussi sensibilité extrême et imagination féconde, écriture et musique, danse qui libère, femme qui délivre. Difficultés à être au monde et linéaments d'une conscience - qui n'est plus mauvaise - et qui s'affirme. Moi étouffé et je qui ose se dire. Individualité vacillante qui, peu à peu, se forge. Le jeune homme de vingt-deux ans a-til construit cette identité qui simplement et avec peine s'esquissait dans l'adolescent de onze ans? Martin en a-t-il fini avec les pères? À ces questions pourrait répondre définitivement ? - une troisième nouvelle qui poursuivrait ce récit d'une vie ${ }^{40}$.

\section{NOTES}

1. Pour une analyse plus approfondie de la nouvelle Der Sonntag, an dem ich Weltmeister wurde, voir : Cozic, A. : «Difficiles identités allemandes : la nouvelle de Friedrich Christian Delius Der Sonntag, an dem ich Weltmeister wurde », in: Knopper, F. et Paul, J.-M. (dir.) : L'homme et la cité allemande au XXe siècle. Souffrance et Résistance, Presses Universitaires de Nancy, 2000, p. 313-332.

La présente contribution reprend, en les condensant, certains aspects de l'analyse ci-dessus, l'essentiel du travail étant ici consacré aux liens entre Der Sonntag et Amerikahaus et à une étude de la seconde nouvelle.

2. «Buster! " hatte der Maler Sauerbaum gestern abend gerufen, nachdem Martin wieder einmal zwei, drei Stunden lang fast nichts gesagt hatte. Delius, F.C. : Amerikahaus und der Tanz um die Frauen, Reinbeck bei Hamburg : Rowohlt Verlag, 1997, p. 8. Nouvelle désormais identifiée dans les notes qui suivent par $A H$, suivi du numéro des pages.

3. Wenn ich schon der Einsame und Stumme bin, dann will ich den Einsamen und Stummen spielen und aus meiner Schwäche eine Rolle machen und dann auch nichts sagen, wenn mir einmal etwas zu sagen einfällt : $A H$, p. 8.

4. Auch Buster hat ein starres Gesicht, auch Buster ist schüchtern, er steckt seine Niederlagen regungslos weg. Wieder und wieder neue Anläufe, unermüdlich bis zum Happy-End. Die Frauen liebten Buster, sie liebten Martin nicht : AH, p. 10.

5. [...] die Scheide, die auf den Bildern der Sexualaufklärer reinlich und trocken aussieht und in Wirklichkeit feucht und haarig ist und vielleicht andere Gefahren birgt als ein Messer, schlüpfrig, saugend, verschlingend, diese dunkle, in unbekannte Tiefen ziehende Wunde $[\ldots .$.$] : AH, p. 40$.

So bewahrte sie ihn vor der Schande, als der Versager vor den Frauen zu erscheinen, der er war : AH, p. 58.

6. Bei dir ist es umgekehrt, die Frauen wenden sich ab, wenn du dich näherst. Aber in Wirklichkeit hast du es mit den beiden noch gar nicht probiert, Margret nie angesprochen, vor der Wilden nur Angst gehabt. Soll dir das Bild Mut machen? Soll es dir sagen, daß du es besser machen sollst als dieser Tänzer ? Oder daß du ein Narziß bist und bleibst ? : AH, p. 120. 
7. Wie kannst du sie lieben, wenn du dich nicht einmal entscheiden kannst zwischen beiden, wenn du nichts zu bestellen hast gegen ihre festen Liebhaber, wenn du ihnen den Rückken zukehrst, wenn du allein tanzt ?: AH, p. 120.

8. [...] jeden Tag spürte er den Vorwurf des Kopfs gegen den Körper : du bist zu häßlich, deshalb verliebt sich keine in dich. Der Körper hielt dem Kopf entgegen : du bist zu schüchtern, deshalb verliebt sich keine in dich. Kopf und Körper stritten um die Schuld, beiden mußte er recht geben : AH, p. 10.

9. Was geht in den Köpfen der Mädchen vor? Was sehen sie, falls sie dich einmal mustern, dich vergleichen mit den anderen Kerlen? Was könnten sie gut finden an dir, was könnte sie bestechen, was hast du zu bieten? Was sehen sie dir an? Sehen sie dir den Stotterer an, den Schweiger, den Tölpel ? Sehen sie dir an, daß du lange nicht mehr geküßt hast ? : AH, p. 131.

10. Als müsse er beweisen, daß der Makel des Gesichts der größte sei, legte er die Stirn in Furchen, verzerrte den Mund, zog mit beiden Händen an den Haaren und musterte die Fratze. So hatte er sich und die Pickel während der Pubertät oft angestarrt, die Pubertät war vorbei, selten wuchsen Pickel nach, die Pubertät war nicht vorbei, jeden Tag spürte er den Vorwurf des Kopfs gegen den Körper [...] : AH, p. 9-10.

11. [...] auch wenn der Aussatz nur ein paar Stellen des Körpers bedeckt, du siehst dich nicht gern nackt, schämst dich deiner Nacktheit, als hätte dieser Körper werweißwas für Sünden begangen $[\ldots]:$ : AH, p. 121 .

12. [...] Blut warf ihn um [...] : AH, p. 100. Das Blut, es fiel ihm das Blut wieder ein, die schreckliche Szene mit dem Blut, das ihm aus dem Körper geflossen war beim letzten Versuch, den erlösenden Akt endlich zu vollziehen [...] : AH, p. 139.

13. Nach und nach hatte er begriffen, daß die fröhliche Unberechenbarkeit zu ihren Reizen gehörte, besonders für einen, dem der Großvaterspruch «Fünf Minuten vor der Zeit ist des Preußen Pünktlichkeit » in den Kopf gemeißelt war : AH, p. 18.

14. Wie einst vor dem Ende des Gottesdienstes, wie heute im Theater oder in der Vorlesung wäre es Martin ungehörig vorgekommen, sich nun vor dem Ende der Demonstration davonzustehlen : AH, p. 84.

15. Das Wort Lust wagte er kaum laut auszusprechen, es gehörte ins Minenfeld des Verbotenen, Fleischeslust, Freßlust, Sinnenlust, Wollust : AH, p. 38.

16. Dazu Szenen mit Christus am Kreuz, im Film wurde eine Verfilmung der Kreuzigung gezeigt. Auch dieses Motiv hatte ihn abgestoßen; er hatte es $\mathrm{zu}$ oft gesehen, zu oft "Ohauptvollblutundwunden» gesungen, war zu oft allein gewesen vor den Bildern von Blut, Opfer, Trauer, Verrat, Schmerzen, Kitsch, Tränen, Vatermacht, Sohnesleid : AH, p. 29-30.

17. Er war nicht so frei, die in zwanzig Jahren Erziehung eingepflanzten Gebote, Verbote und Erwartungen Schritt für Schritt abzuschütteln und $\mathrm{zu}$ vergessen. Nach allem, was er widerstrebend gelernt hatte, gehörte es sich nicht, mitten auf der Fahrbahn zu laufen und den Verkehrsfluß zu stören, gehörte es sich noch weniger, für ein politisches Ziel auf die Straße zu gehen und eine kritische Meinung zu einem lästigen Krieg in die friedliche Berliner Innenstadt $\mathrm{zu}$ tragen und gegen "unsere Freunde und Beschützer» aufzutreten. Die hemmenden Erziehungsreste ließen sich nicht völlig aus dem Kopf räumen. Trotz des sich entwickelnden Eigensinns blieb er in den Netzen alter Verbote gefangen : AH, p. 62.

18. Immer wieder fragte sich Martin, wieviel er von den Eltern geerbt hatte. Er wußte es nicht, er wagte nicht, Lust und Sünde auszuprobieren, aus Angst vor dem abgewürgten Gott in ihm, Angst vor dem toten Vater in ihm, Angst vor der ängstlichen Mutter in ihm, und er haßte sich für diese Ängste : AH, p. 38.

19. Sie stecken in dir mit ihren Sprüchen und strengen Augen, du bist ihr Fleisch, ihr Blut, ihr Gedanke, und in deinen Adern, in deinen Nerven spürst du, was sie gut und was sie verwerflich finden, was verboten ist und ans Verbotene grenzt. "Gerade die sexuellen Dinge ", hat der Großvater geschrieben, «sind heute in verhängnisvoller Weise hochgespielt worden und 
entfalten eine dämonische Macht. Nur sich nicht treiben lassen, nur nicht sich vom Zeitgeist bestimmen lassen, nur nicht die warnende Stimme des Gewissens überhören » : AH, p. 109-110.

20. Seine Vernunft kämpfte gegen die eingefleischten Kindergedanken : Alles wird bestraft, Gott sieht alles, die Eltern sehen fast alles, überall lauern Sünden, und am schlimmsten ist die Strafe für das, was man gar nicht getan hat aus Angst vor der Strafe. Bevor das Kind tut, was als böse gilt, sieht es sich schon verurteilt : AH, p. 39.

21. Das Messer, immer bleibt das Messer in der Luft, das auf den Jungen gerichtete Messer. Das dir die Zunge abgeschnitten hat, das dich stumm gemacht hat. Das Messer, was schneidet das Messer, was kann man dir abschneiden, das Glied schneiden sie dir ab, warum sagten sie, du bist ein «Glied der Kirche », seit du getauft und konfirmiert bist, warum schämten sie sich nicht, das gleiche Wort wie für das Geschlechtsteil zu benutzen, als wäre dein Glied schon vergeben, das Sündenkörperteil der Christenheit geopfert, abgeschnitten, weg : AH, p. 40.

22. Die andern hielten ihn nicht auf, sie kannten ihn als den, der wenig sagt, wenig trinkt, früh geht : AH, p. 36. [...] Versager vor den Frauen : AH, p. 58. Im übrigen war es völlig unwichtig, was er, der stumme Student, der linkische Demonstrant, der hilflos Verliebte über Fellini sagte oder dachte : $A H$, p. 81.

23. Sie haben dich Martin getauft, der ganze dicke Luther eine unsichtbare Last auf deinen Schultern, ein gefährlicher Flüsterer, der dir mit seinem großen und kleinen Katechismus die Ohren vollwispert: Tu's nicht, tu's nicht! Bild dir nicht ein, die elterlichen Autoritäten hätten keine Macht mehr über dich : AH, p. 109.

24. Er wollte nichts falsch machen. Er fürchtete Strafen. Er wollte nicht schuldig werden. Er sah keinen Sinn darin, Widerstand zu leisten gegen die Staatsgewalt. Unschuldig wollte er sein an den Eierwürfen und am Fahnenzerren und der Prügelei, nicht schuldig sein durch Schweigen zum mörderischen Krieg. Er suchte eigensinnig zu sein und wollte ihn lässig aushalten, den Widerspruch : sich einordnen in die Demonstration und bei Gefahr wieder heraustreten und den kritischen Beobachter spielen : AH, p. 100-101.

25. Warum dich nicht verlocken lassen zu Ausschreitungen, antiamerikanischen, antiväterlichen, antimütterlichen, antigroßväterlichen, antichristlichen Ausschreitungen ? : AH, p. 110.

26. Wer nicht mehr geradeaus schreitet, nicht nach Befehl marschiert, wer die Kontrolle verliert, wer aus der Rolle fällt, wer Krach macht und rebelliert, begeht eine Ausschreitung oder was? Wann wird welche Tat eine Ausschreitung, wo fängt es an mit den Ausschreitungen?: $A H$, p. 152-153.

27. Was für ein Krieg, über den man noch dankbar sein muß, weil es dem Vater besser ergangen ist als dessen Vater, der gleich in den ersten Tagen des Ersten Weltkrieges den Fangschuß erhielt. Und der Krieg im Nachkrieg, als die Erwachsenen die Vergangenheit hinunterwürgten, allen voran der Großvater [...] : AH, p. 54.

28. Warum bildest du dir das ein, warum Gefühle für Vietnam, aber viel weniger bei den anderen Kriegen ? Weil du gegen die USA bist? Nein, weil jeden Morgen die Zeitung eine Reaktion von dir verlangt : Hinnehmen oder Aufregen, Abstumpfen oder Wachwerden. Weil es Gut und Böse gibt, weil es einfach ist, diesen Krieg abzulehnen. Weil alle vernünftigen Leute dagegen sind [...] : $A H$, p. 53.

29. Er war nicht gewohnt, mitten in einer Menge zu gehen. Nicht gewohnt, mitten auf der Fahrbahn zu gehen, erst recht nicht mitten in der City. Er war mittendrin und fühlte sich am falschen Platz: AH, p. 60. Martin wußte nicht, ob jemals so viele Augen auf ihn geschaut hatten. Das Angenehme war, daß keiner ihn als Person sah oder ihn meinte, er war Teil dieser langsam laufenden Menge und geschützt in ihr. Durch die Blicke der Zuschauer ringsum wurde er zu einem Demonstranten wie die anderen auch, endlich konnte er seine innere Zögerlichkeit ablegen. Es gefiel ihm, versteckt und gleichzeitig beteiligt zu sein : AH, p. 70 .

30. Jedes Wort mußte, bevor es über die Lippen kam, auf seine Sprechbarkeit hin überprüft werden. In Hunderstel oder Zehntel Sekunden hatte das Hirn zu entscheiden, ob das Wort, das 
sich im Sprachzentrum aufdrängte und nach dessen Sinn der gerade entstehende Satz oder Gedanke verlangte, mit einem Verschlußlaut wie B, C, D, G, P, Q, T, oder Z begann und ob das Wagnis trotz des zu erwartenden Hindernisses eingegangen, mit vielleicht glückender, vielleicht mißglückender Verschiebung der Betonung überspielt oder schnell ein Ersatzwort gesucht werden sollte, das mit einem Vokal oder mit sprechfreundlichen Nasalen oder Reibelauten anfing : $A H$, p. 24.

31. War ein Wort wie «klein» nicht zu vermeiden, mußte es je nach Zusammenhang in ein reibungsloses, vielleicht weniger treffendes übersetzt werden, «schmal, winzig, unbedeutend » $[\ldots .]:$. : AH, p. 25.

32. Alle Energie richtete Martin, weil er vergessen wollte, daß er stumm war, auf die Sprache. An gedruckten Wörtern berauschte er sich ebenso wie an selber geschriebenen, er zerlegte une analysierte, was ihm den Rausch verschaffte, und flog von einem Buch zum andern, süchtig nach Phantasie und Witz, nach klaren, wilden Sätzen [...] : AH, p. 11.

33. Beim Schreiben sah ihm keiner über die Schulter, kein Gott, kein Vater, kein Großvater, auch nicht die Mutter. Schreibend fühlte er sich autonom, allein in einem Geheimbund mit den Wörtern oder in einem freundschaftlichen Zweikampf, und er war sicher, am Ende gegen sie nicht verlieren zu können : $A H$, p. 44.

34. Auffallen wollte er, wenigstens mit ein paar originellen Sätzen [...] : AH, p. 42.

35. Jeder Satz war auf Beifall aus, und Martin spürte das, aber es störte ihn nicht, es war die einzige Möglichkeit, Aufmerksamkeit und Sympathie zu gewinnen, vielleicht sogar bei den wenigen Frauen im Seminar : AH, p. 47.

36. Wo liegt der Fehler? «Leib». Warum das altmodisch, christlich geprägte Wort, warum nicht « Körper»? Also weg mit « Leib », dem Vaterwort, Fremdwort ! Es mußte gemieden werden, weil es die Suche nach der eigenen Sprache sabotierte. Diese Wörter vergifteten ihn, lähmten ihn [...] : AH, p. 117.

37. Im Wippen und Steppen, im Zucken und Schwingen schüttelte er unsichtbare Krücken ab, stampfte seine Verlorenheit nieder, mit Beugen und Schwenken des Beckens versuchte er das Bild zu wiederlegen, das er von sich hatte, das Bild des einsamen, in sich gekehrten Tänzers, das Kirchner-Bild, das Bild der Erstarrung, das sich widerlegen ließ, wenn er nur auf die Musik hörte und die Frau im Auge behielt: AH, p. 134.

38. « Do you speak English? » fragte sie. «Yes, but... not very well» : AH, p. 135.

39. Du hast gewonnen, sagte er sich, gewonnen, nicht etwa verloren, die sogenannte Unschuld, wie lächerlich, nein, deine Schuld hast du verloren, deine Schande verloren, hast gewonnen, gewonnen! : AH, p. 149.

40. Resterait à analyser, à propos de Der Sonntag comme de Amerikahaus, l'aspect fortement autobiographique de ces deux nouvelles, si l'on en croit en tout cas les propres déclarations de Delius. Ainsi dans un entretien avec Keith Bullivant en avril 1994 aux États-Unis : "Wer Der Sonntag [...] gelesen hat, weiß, wie schwer ich es hatte, buchstäblich zur Sprache zu kommen und das persönliche Sprechen zu entwickeln» (Bullivant, K. : «Bewundernswert, was Sie alles schon damals [...]. Gespräch mit Friedrich Christian Delius». In : Literatur für Leser 95/1, p. 1). Ou encore : «In Der Sonntag [...] habe ich ja deutlich genug beschrieben, wie ich aufgewachsen bin in einer Familie, in einem Haus, in dem alles am Wort gemessen wurde. Das Wort Gottes bestimmte auch die Worte des Alltags, alles war positiv oder negativ auf die vom Vater verkündeten und von der Mutter gesungenen Bibel- und Glaubensworte bezogen. Ich lebte in einem Wortkäfig, der mich in jeder Beziehung unterdrückte, also entwickelte ich früh ein Mißtrauen gegen die vorgegebenen, von oben verordneten Wörter [...]» (Ibid., p. 8). Propos repris dans un autre entretien avec le même Keith Bullivant en mai 1996 à Berlin (Bullivant, K. : « Das könnte dein Schreiben sein ». Gespräch mit Friedrich Christian Delius. In: Durzak, M. und Steinecke, H. (hrsg.) : F.C. Delius. Studien über sein literarisches Werk, Tübingen: Stauffenburg Verlag, 1997, p. 225-241). 


\section{RÉSUMÉS}

Amerikahaus und der Tanz um die Frauen (1997) est la suite de la nouvelle écrite par F.C. Delius en 1994, Der Sonntag, an dem ich Weltmeister wurde. La présente contribution a pour objectif d'analyser l'étroite corrélation entre les deux œuvres.

Ce « dimanche »-là, le 4 juillet 1954, si l'équipe nationale de football de la RFA, grâce à sa victoire en finale sur la Hongrie, conquiert le titre de championne du monde, elle permet aussi à la nation allemande, moralement et politiquement affaiblie, d'acquérir de nouveau une certaine notoriété. Ce «dimanche »-là aussi, et surtout, le narrateur de la nouvelle, adolescent de onze ans à l'époque, fils de pasteur, parvient, en écoutant à la radio, fasciné par les paroles du reporter, la retransmission de la finale, à s'échapper un temps de l'univers familial où règne la figure toute puissante du père, elle-même émanation du Tout-Puissant, à briser la « cage du père ", la « cage de mots » où d'ordinaire il se sent enfermé.

Si Amerikahaus montre l'éveil d'une conscience politique et littéraire et l'initiation sexuelle de celui qui est désormais un étudiant de vingt-deux ans - Martin participe à Berlin, devant la "Maison de l'Amérique ", à une manifestation contre la guerre du Vietnam, écrit des poèmes, "danse autour des femmes»-, les nombreuses réflexions, remarques, auto-analyses psychologiques, bribes de souvenirs de ce jeune homme toujours timide, complexé, hésitant, bafouillant, prouvent aussi qu'il n'est guère aisé d'« en finir avec les pères ", d'échapper définitivement à l'ordre familial, de se libérer des principes, commandements et interdits qui furent un jour inculqués.

Amerikahaus und der Tanz um die Frauen (1997) ist die Fortsetzung der von F.C. Delius 1994 verfaßten Erzählung Der Sonntag, an dem ich Weltmeister wurde. Ziel der vorliegenden Arbeit ist, die enge Verbindung zwischen den beiden Novellen aufzuzeigen.

An diesem «Sonntag ", dem 4. Juli 1954, hat die Fußballnationalmannschaft der Bundesrepublik mit ihrem Sieg über Ungarn nicht nur den Weltmeistertitel erspielt, sondern auch der moralisch und politisch angeschlagenen Nation eine Art - allerdings noch beschränktes - Selbstwertgefühl wiedergegeben. An diesem «Sonntag " gelingt es auch und vor allem dem elfjährigen Erzähler und Pfarrersohn, der der Endspielübertragung im Rundfunk lauschen darf und den die Worte des Reporters faszinieren, vorläufig aus dem evangelischen Pfarrhaus, wo der wortgewaltige, allmächtige Vater, Stellvertreter des Allmächtigen, herrscht, aus dem «Vaterkäfig », aus dem « Wortkäfig » auszubrechen.

Auch wenn Amerikahaus das Erwachen des politischen und literarischen Bewußtseins sowie die sexuelle Initiation des nun zweiundzwanzigjährigen Studenten zeigt - Martin nimmt in Berlin vor dem Amerikahaus an einer Demonstration gegen den Krieg in Vietnam teil, schreibt Gedichte, tanzt um die Frauen -, so beweisen auch die vielen Reflexionen, Bemerkungen, psychologischen Selbstanalysen, Erinnerungssplitter des immer noch schüchternen, verklemmten, zögernden, stotternden jungen Mannes, daß es ihm nicht leicht fällt, die Väter zu vergessen, auf die Väter zu verzichten, sich von der puritanischen Ordnung des Elternhauses, von den einmal eingetrichterten Prinzipien, Geboten und Verboten endgültig zu befreien.

\section{AUTEUR}

\section{ALAIN COZIC}

Université de Toulouse-Le Mirail 